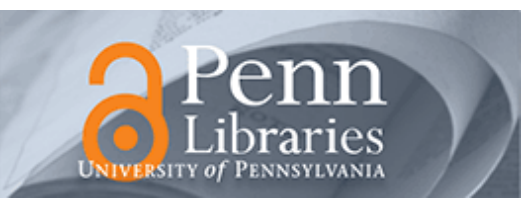

University of Pennsylvania

ScholarlyCommons

March 2004

\title{
High Impedance Metamaterial Surfaces Using Hilbert-Curve Inclusions
}

\author{
John McVay \\ Villanova University
}

Nader Engheta

University of Pennsylvania, engheta@seas.upenn.edu

Ahmad Hoorfar

Villanova University

Follow this and additional works at: https://repository.upenn.edu/ese_papers

\section{Recommended Citation}

John McVay, Nader Engheta, and Ahmad Hoorfar, "High Impedance Metamaterial Surfaces Using HilbertCurve Inclusions", . March 2004.

Copyright 2004 IEEE. Reprinted from IEEE Microwave and Wireless Components Letters, Volume 14, Issue 3, March 2004, pages 130-132.

Publisher URL: http://ieeexplore.ieee.org/xpl/tocresult.jsp?isNumber=28582\&page=1

This material is posted here with permission of the IEEE. Such permission of the IEEE does not in any way imply IEEE endorsement of any of the University of Pennsylvania's products or services. Internal or personal use of this material is permitted. However, permission to reprint/republish this material for advertising or promotional purposes or for creating new collective works for resale or redistribution must be obtained from the IEEE by writing to pubs-permissions@ieee.org. By choosing to view this document, you agree to all provisions of the copyright laws protecting it.

This paper is posted at ScholarlyCommons. https://repository.upenn.edu/ese_papers/6

For more information, please contact repository@pobox.upenn.edu. 


\title{
High Impedance Metamaterial Surfaces Using Hilbert-Curve Inclusions
}

\author{
Abstract \\ A metamaterial surface, composed of a periodic arrangement of Hilbert Curve inclusions above a \\ conducting ground plane, is analyzed numerically and is shown to possess the properties of a high \\ impedance surface by investigating the phase and magnitude of the reflection coefficient, $\Gamma$, for a plane \\ wave of normal incidence. A parametric study is presented with respect to the iteration order of the \\ Hilbert curve, the surface height above the ground plane, and the separation distance between the \\ neighboring Hilbert elements within the surface array.

\section{Keywords} \\ Artificial magnetic conductor, complex surface, high impedance ground plane, Hilbert curve, metamaterial

\section{Comments} \\ Copyright 2004 IEEE. Reprinted from IEEE Microwave and Wireless Components Letters, Volume 14, Issue \\ 3, March 2004, pages 130-132. \\ Publisher URL: http://ieeexplore.iee. org/xpl/tocresult.jsp?isNumber=28582\&page=1 \\ This material is posted here with permission of the IEEE. Such permission of the IEEE does not in any way \\ imply IEEE endorsement of any of the University of Pennsylvania's products or services. Internal or \\ personal use of this material is permitted. However, permission to reprint/republish this material for \\ advertising or promotional purposes or for creating new collective works for resale or redistribution must \\ be obtained from the IEEE by writing to pubs-permissions@ieee.org. By choosing to view this document, \\ you agree to all provisions of the copyright laws protecting it.
}




\title{
High Impedance Metamaterial Surfaces Using Hilbert-Curve Inclusions
}

\author{
John McVay, Nader Engheta, Fellow, IEEE, and Ahmad Hoorfar, Senior Member, IEEE
}

\begin{abstract}
A metamaterial surface, composed of a periodic arrangement of Hilbert Curve inclusions above a conducting ground plane, is analyzed numerically and is shown to possess the properties of a high impedance surface by investigating the phase and magnitude of the reflection coefficient, $\Gamma$, for a plane wave of normal incidence. A parametric study is presented with respect to the iteration order of the Hilbert curve, the surface height above the ground plane, and the separation distance between the neighboring Hilbert elements within the surface array.
\end{abstract}

Index Terms-Artificial magnetic conductor, complex surface, high impedance ground plane, Hilbert curve, metamaterial.

\section{INTRODUCTION}

H IGH impedance surfaces, also known as artificial magnetic conductors, have received considerable attention in the last few years [1]-[6]. These surfaces have a reflection coefficient of $\Gamma \simeq+1$, when illuminated with a plane wave, instead of the typical $\Gamma \simeq-1$ for a conventional perfectly electric conducting (PEC) surface. These structures can obviously offer interesting applications in the antenna design [1]-[5] and for thin absorbing screens [6]. For example a dipole antenna above such a metamaterial surface will have an image current with the same phase as the current on the dipole, resulting in an enhanced radiation performance. Several different types of high-impedance ground planes have been studied by various research groups (see, e.g., [1]-[6]).

The Hilbert curve, first proposed by Hilbert in 1891, is a member of the family of curves known in the mathematics literature as the "space-filling curves," the first of which was introduced in 1890 by Peano [7]. The Hilbert curve offers certain attractive properties, since a structure of this shape can be made of an electrically long metallic wire compacted within a very small footprint. Moreover, this space-filling geometry can be a planer structure, thus allowing for ease of fabrication. As the iteration order of the curve increases, the Hilbert Curve may maintain its footprint size, while the length of curve increases. This property is what allows the Hilbert curve to posses a relatively low resonant frequency, i.e., a long resonant wavelength with respect to the linear dimension of its footprint. The Hilbert curve geom-

\footnotetext{
Manuscript received July 1, 2003; revised October 27, 2003. This work was supported in part by DARPA under Grant MDA972-02-1-0022. The content of this letter does not necessarily reflect the position or the policy of the U.S. Government, and no official endorsement should be inferred. The review of this letter was arranged by Associate Editor Rüdiger Vahldieck.

J. McVay and A. Hoorfar are with Department of Electrical and Computer Engineering, Villanova University, Villanova, PA 19085 USA.

N. Engheta is with Department of Electrical and Systems Engineering, University of Pennsylvania, Philadelphia, PA 19104 USA.

Digital Object Identifier 10.1109/LMWC.2003.822571
}

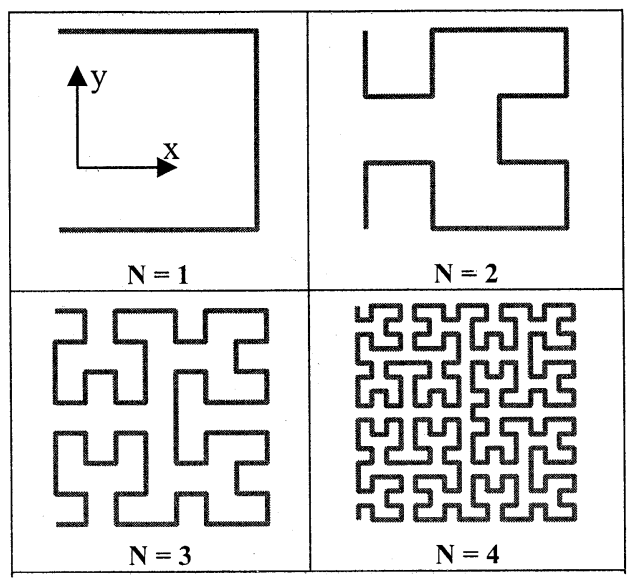

Fig. 1. Hilbert curves with various iteration order number N.

etry has been used in the small antenna design [8]-[10] and also for the frequency-selective surfaces (e.g., [11]).

In the present study, we explore the possibility of having a metamaterial surface in which many inclusions in the shape of the "Hilbert curve" are placed, in a two-dimensional (2-D) periodic arrangement, on a host surface. Some preliminary results of our work were presented in a recent symposium [12].

\section{RESONANCES FOR A Single Hilbert INCLUSION}

Before we analyze the behavior of a metamaterial surface made of periodic arrangement of Hilbert curve inclusions above a PEC ground plane, we investigate numerically the resonance behavior of a single Hilbert curve as a scatterer. Using a method of moments (MoM) numerical code, ${ }^{1}$ we simulate a single Hilbert curve inclusion of varying iteration orders in free space, made of a PEC wire with radius $0.01 \mathrm{~mm}$, in order to determine the resonant frequencies of the Hilbert Curve structure for each iteration order. The single Hilbert curve is assumed to have $1.2 \mathrm{~mm} \times 1.2 \mathrm{~mm}$ footprint. A normally incident plane wave with two different orthogonal polarizations, i.e., $E_{x}$ or $E_{y}$, illuminates this Hilbert curve, shown in Fig. 1, and the induced current along the wire in the Hilbert curve is evaluated and its maximum value is determined. Then the frequency of the incident wave is varied, and the variation of this maximum value as a function of frequency is obtained. The frequency at which this maximum value reaches its highest value (i.e., the "maximum of maxima"), we name the resonant frequency $F_{\mathrm{MAX}}$. Linear interpolation was then utilized in order to find the frequencies at which this maximum current falls to 0.707

${ }^{1}$ GNEC, Nittany Scientific, Inc., ver. 1.4 
TABLE I

RESONANCES, Fractional BANDWIDTHS, AND SidE DimENSIONS (WITH RESPECT TO THE RESONANT WAVELENGTHS, $\lambda$ ) FOR SiNGLE HILBERT-CURVE SCATTERERS WITH VARIOUS ITERATION ORDER N

\begin{tabular}{l|c|c|c}
\hline Hilbert Order \# & $\mathbf{N = 1}$ & $\mathbf{N}=\mathbf{2}$ & $\mathbf{N = 3}$ \\
\hline$f_{\max }$ & 87.4 & 58.4 & 40.6 \\
$\mathbf{E}_{\mathbf{X}}$ Excitation (GHz) & & & \\
\hline$f_{\max }$ & 41.6 & 29.1 & 20.7 \\
$\mathbf{E}_{\mathbf{Y}}$ Excitation(GHz) & & & \\
\hline$\frac{\Delta f}{\frac{f_{\max }}{\Delta f} \text { for } \mathbf{E}_{\mathbf{X}}}$ & $18.79 \%$ & $9.41 \%$ & $3.5 \%$ \\
\hline$\frac{\Delta f}{f_{\max }}$ for $\mathbf{E}_{\mathbf{Y}}$ & $5.55 \%$ & $1.39 \%$ & $0.5 \%$ \\
$\begin{array}{l}\text { Side Dimension for } \\
\mathbf{E}_{\mathbf{X}}\end{array}$ & $0.35 \lambda$ & $0.23 \lambda$ & $0.16 \lambda$ \\
\hline $\begin{array}{l}\text { Side Dimension for } \\
\mathbf{E}_{\mathbf{Y}}\end{array}$ & $0.17 \lambda$ & $0.12 \lambda$ & $0.08 \lambda$ \\
\hline
\end{tabular}

of its maximum of maxima, and the corresponding fractional bandwidth was calculated. The results for the Hilbert curve element with various iteration orders shown in Fig. 1, are listed in Table I. As can be seen from this table, as the iteration order is increased, the resonant frequency and the fractional bandwidth are decreased for both polarizations of the normally incident plane wave. The last two rows on the bottom indicate the relative linear (side) dimension of the footprint of the Hilbert curve with respect to its resonant wavelength $\lambda$ in each iteration order. A higher iteration order for the Hilbert curve provides an electrically smaller element. We also note from Table I that the resonant frequency, fractional bandwidth, and the relative side dimensions for the $x$-polarized plane wave excitation are larger than those for the $y$-polarized illumination. This is due to the presence of the mirror symmetry of the Hilbert curve with respect to the $x-z$ plane, which leads to the odd and even symmetric distributions of the induced current along the wire for the $x$-polarized and $y$-polarized excitations, respectively.

\section{Hilbert ARRAY SURFACE}

A surface can be conceptually constructed utilizing the Hilbert curve inclusions by creating a periodic, 2-D array of Hilbert elements, located in parallel with, and at a small distance above, a PEC ground plane as is shown in Fig. 2. To model the wave interaction with this surface numerically, we use the periodic MoM with periodic boundary conditions in the software package IE3D code. ${ }^{2}$ The inter-element edge to edge spacing between any two neighboring Hilbert inclusions in the surface array is chosen to be equal to the length of a single section of the Hilbert Curve, which varies with the iteration order of the Hilbert Curve as $d=L /\left(2^{N}-1\right)$ with $L$ being the linear (side) dimension of the Hilbert curve footprint.

Fig. 3 shows the magnitude and phase of the reflection coefficient $\Gamma$, for an $x$-polarized normal incidence plane wave excita-

2IE3D, Zeland Software, Inc., ver. 9.3

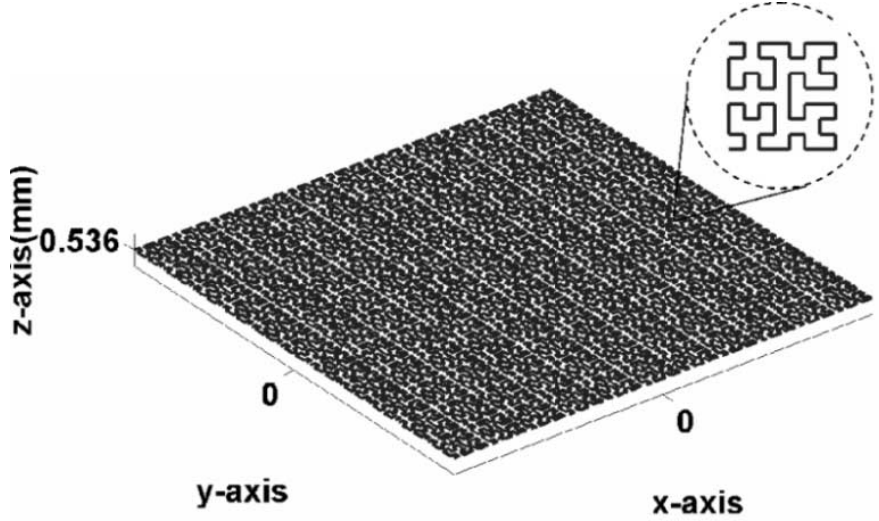

Fig. 2. Hilbert surface of order 3 made of 2-D period arrangement of Hilbert inclusions of order 3 above a PEC ground plane.
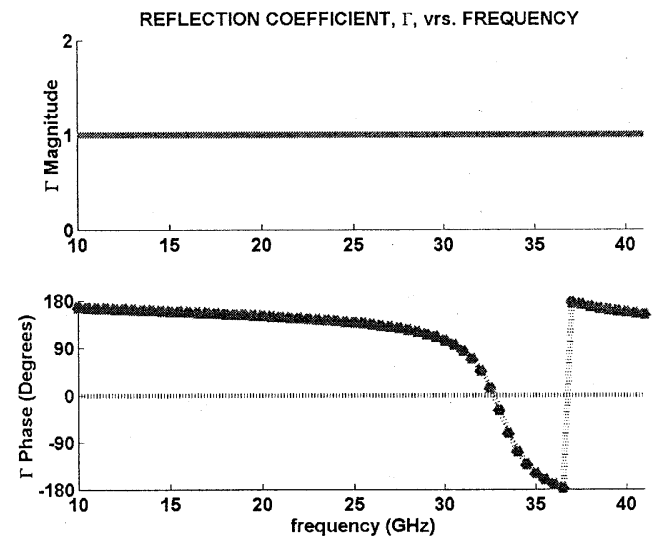

Fig. 3. Magnitude and phase of the reflection coefficient for an $x$-polarized normally incident plane wave illuminating a Hilbert surface with order $\mathrm{N}=3$ at a distance $0.536 \mathrm{~mm}$ above a PEC ground plane.

tion, as a function of the frequency, for the Hilbert surface made of Hilbert inclusions of order $\mathrm{N}=3$ at a height of $0.536 \mathrm{~mm}$ above a PEC ground plane. The phase of this reflection coefficient is evaluated with respect to the top of the Hilbert surface. As can be seen from this figure, a reflection coefficient of $\Gamma=+1$ (where the phase of $\Gamma$ is zero) is achieved at a frequency of about $32.7 \mathrm{GHz}$, which we denote $F_{\text {HIS }}$ with the subscript "HIS" standing for "high-impedance surface". Table II presents the results of our parametric study on the role of iteration orders of the Hilbert curves and the height of the surface above the ground plane. The value $\Delta \mathrm{f}$, is chosen as the $\pm 90^{\circ}$ crossings for the phase of $\Gamma$, although other values for such crossings can be considered [5]. The bandwidth of the structure decrease as the iteration order $\mathrm{N}$ increases and/or the height $h$ decreases.

The results of our parametric study on the effect of separation distance between the adjacent Hilbert elements on the performance of the Hilbert surface are shown in Table III. We choose the separation distance to be $5 d, 2 d, 0.5 d$, and $0.2 d$, where $d$ is the original separation distance of $0.171 \mathrm{~mm}$, for the case of a Hilbert surface of order 3 at a height of $1.072 \mathrm{~mm}$ above a PEC ground plane. We note how little $F_{\mathrm{HIS}}$ and the bandwidths vary with this separation distance, while they vary more considerably when the iteration order and the height change. In our simulation here, we have assumed the air spacing between the Hilbert 
TABLE II

VARIATION OF FREQUENCY $F_{\text {HIS }}$ AT WHICH $\Gamma=+1$, AND THE FRACTIONAL BANDWIDTH OF THE HILBERT SURFACE IN TERMS OF THE ITERATION ORDER AND the HeIGHT ABOve THE PEC GROUND Plane

\begin{tabular}{|c|c|c|c|c|}
\hline $\begin{array}{l}\text { Hilbert } \\
\text { Order \# }\end{array}$ & $\begin{array}{l}\text { Frequency } \\
\text { (Ex) }\end{array}$ & $\begin{array}{l}\text { Frequency } \\
\text { (EY) }\end{array}$ & $\frac{\Delta f}{f_{H I S}}$ & $\frac{\Delta f}{f_{H I S}}$ \\
\hline \multicolumn{5}{|c|}{ Height Above Ground Plane $=0.536 \mathrm{~mm}$} \\
\hline $\mathrm{N}=1$ & $77.23 \mathrm{GHz}$ & $41.08 \mathrm{GHz}$ & $31.94 \%$ & $5.50 \%$ \\
\hline $\mathrm{N}=2$ & $48.54 \mathrm{GHz}$ & $27.37 \mathrm{GHz}$ & $18.90 \%$ & $3.01 \%$ \\
\hline $\mathrm{N}=3$ & $32.66 \mathrm{GHz}$ & $18.17 \mathrm{GHz}$ & $9.09 \%$ & $1.95 \%$ \\
\hline \multicolumn{5}{|c|}{ Height Above Ground Plane $=1.072 \mathrm{~mm}$} \\
\hline $\mathrm{N}=1$ & $57.87 \mathrm{GHz}$ & $38.54 \mathrm{GHz}$ & $77.71 \%$ & $29.8 \%$ \\
\hline $\mathrm{N}=2$ & $40.10 \mathrm{GHz}$ & $26.25 \mathrm{GHz}$ & $50.51 \%$ & $13.3 \%$ \\
\hline $\mathrm{N}=3$ & $28.87 \mathrm{GHz}$ & $17.61 \mathrm{GHz}$ & $29.64 \%$ & $5.49 \%$ \\
\hline \multicolumn{5}{|c|}{ Height Above Ground Plane $=2.144 \mathrm{~mm}$} \\
\hline $\mathrm{N}=1$ & $31.13 \mathrm{GHz}$ & $28.95 \mathrm{GHz}$ & $128.49 \%$ & $52.8 \%$ \\
\hline $\mathrm{N}=2$ & $27.04 \mathrm{GHz}$ & $22.90 \mathrm{GHz}$ & $89.70 \%$ & $47.6 \%$ \\
\hline $\mathrm{N}=3$ & $22.08 \mathrm{GHz}$ & $16.35 \mathrm{GHz}$ & $65.24 \%$ & $25.4 \%$ \\
\hline
\end{tabular}

TABLE III

VARIATION OF $F_{\text {HIS }}$ AND THE BANDWIDTH IN TERMS OF THE SEPARATION DISTANCE

Hilbert Surface of Order 3 at Height $1.072 \mathrm{~mm}$ Above Conducting Ground Plane

\begin{tabular}{l|l|l|l|l}
\hline $\begin{array}{l}\text { Inter- } \\
\text { Element } \\
\text { Spacing }\end{array}$ & $(\mathrm{Ex})$ & $(\mathrm{Ey})$ & $\begin{array}{l}\text { Bandwidth } \\
(\mathrm{Ex})\end{array}$ & $\begin{array}{l}\text { Bandwidth } \\
(\mathrm{Ey})\end{array}$ \\
\hline $0.034 \mathrm{~mm}$ & $24.44 \mathrm{GHz}$ & $15.65 \mathrm{GHz}$ & $26.88 \%$ & $7.15 \%$ \\
\hline $0.085 \mathrm{~mm}$ & $26.72 \mathrm{GHz}$ & $16.82 \mathrm{GHz}$ & $28.77 \%$ & $6.83 \%$ \\
\hline $0.171 \mathrm{~mm}$ & $28.87 \mathrm{GHz}$ & $17.61 \mathrm{GHz}$ & $29.64 \%$ & $5.49 \%$ \\
\hline $0.342 \mathrm{~mm}$ & $31.46 \mathrm{GHz}$ & $18.23 \mathrm{GHz}$ & $29.38 \%$ & $4.82 \%$ \\
\hline $0.855 \mathrm{~mm}$ & $35.16 \mathrm{GHz}$ & $19.01 \mathrm{GHz}$ & $26.17 \%$ & $3.42 \%$ \\
\hline
\end{tabular}

surface and the ground plane. If a dielectric layer is used instead, one can anticipate that the resonant frequencies will be lowered.

\section{SUMMARY}

By numerically investigating the reflection coefficient of a normally incident plane wave on a surface conceptually constructed with 2-D periodic arrangement of Hilbert curve inclusions above a conducting ground plane, we have shown that this surface can act as a high impedance surface within a certain frequency band, providing a reflection coefficient of magnitude 1 with a phase angle of $0^{\circ}$. The frequency at which this surface becomes a high-impedance surface is primarily related to the order number of the Hilbert curve (i.e., the length of the curve). This frequency, as well as the bandwidth associated with its range of operation, is affected by the height of the surface above the ground plane and to the lesser extent by the distance of separation between adjacent Hilbert elements within the array. Such a structure as a high-impedance surface can have interesting applications in the antenna design. Such applications are presently under investigation [12] and will be reported in detail in a future paper.

\section{REFERENCES}

[1] D. Sievenpiper, L. Zhang, R. F. J. Broas, N. G. Alexopolous, and E. Yablonovitch, "High-impedance electromagnetic surfaces with a forbidden frequency band," IEEE Trans. Microwave Theory Tech., vol. 47, pp. 2059-2074, Nov. 1999

[2] F. Yang and Y. Rahmat-Samii, "Reflection phase characterization of an electromagnetic band-gap (EBG) surface," in Dig. 2002 IEEE Antennas and Propagation Soc. Int. Symp., vol. 3, San Antonio, TX, June 16-21, 2002, pp. 744-747.

[3] Y. Zhang, J. von Hagen, and W. Wiesbeck, "Patch array as artificial magnetic conductors for antenna gain improvement," Microw. Opt. Technol. Lett., vol. 35, no. 3, pp. 172-175, Nov. 5, 2002.

[4] R. Gonzalo, P. de Maagt, and M. Sorolla, "Enhanced patch-antenna performance by suppressing surface waves using photonic-bandgap substrates," IEEE Trans. Microwave Theory Tech., vol. 47, pp. 2131-2138, Nov. 1999.

[5] R. C. Hansen, "Effects of a high-impedance screen on a dipole antenna," IEEE Antennas Wireless Propagat. Lett., vol. 1, pp. 46-49, 2002.

[6] N. Engheta, "Thin absorbing screens using metamaterial surfaces," in Dig. 2002 IEEE AP-S Int. Symp., vol. 2, San Antonio, TX, June 16-21, 2002, pp. 392-395.

[7] H. Sagan, Space-Filling Curves. New York: Springer-Verlag, 1994.

[8] K. J. Vinoy, K. A. Jose, V. K. Varadan, and V. V. Varadan, "Hilbert curve fractal antenna: A small resonant antenna for VHF/UHF applications," Microw. Opt. Tech. Lett., vol. 29, no. 4, pp. 215-219, May 2001.

[9] S. R. Best, "A comparison of the performance properties of the Hilbert curve fractal and meander line monopole antennas," Microw. Opt. Tech. Lett., vol. 35, no. 4, pp. 258-262, Nov. 20, 2002.

[10] J. Zhu, A. Hoorfar, and N. Engheta, "Bandwidth, cross polarization, and feed-point characteristics of matched Hilbert antennas," IEEE Antennas Wireless Propagat. Lett., vol. 2, no. 1, pp. 2-5, 2003.

[11] E. A. Parker and A. N. A. El Sheikh, "Convoluted array elements and reduced size unit cells for frequency-selective surfaces," Proc. Inst. Elect. Eng. Part H: Microwaves, Antennas, and Propagation, vol. 138, pp. 19-22, Feb. 1991.

[12] J. McVay, A. Hoorfar, and N. Engheta, "Applications of Hilbert artificial magnetic conductor ground planes to performance enhancement of electrically small antennas," in Proc. 2003 IEEE Sarnoff Symp. Advanced Wired and Wireless Communications, Mar. 11-12, 2003, pp. 297-300. 